\title{
Expanding understandings of wellbeing through researching women's experiences of intergenerational somatic dance classes.
}

\author{
Karen Barbour, Marianne Clark and Allison Jeffrey
}

\begin{abstract}
Engaging a feminist ethnographic methodology, this article offers a discussion of women's embodied experiences of wellbeing in intergenerational somatic dance classes. Somatic dance classes aim to develop embodied awareness, support ease and freedom in movement, and offer opportunities for creativity, agency and reflection. Drawing on in-depth interviews, observation and autoethnographic vignettes, three themes emerged from the empirical material that expand understandings of wellbeing as a fluid and dynamic experience, reveal the value for women in moving for movement's sake, and identify the significance of intergenerational contexts for moving together. As a consequence, this research offers insight into ways in which women participating in somatic dance classes have re-interpreted wellbeing practices, 're-claiming' wellbeing from circulating neoliberal, self-improvement and productivity agendas, and instead dancing into wellbeing.
\end{abstract}

\section{Introduction}

Wellbeing is a growing area of research, interdisciplinary collaboration, academic discussion and debate (Dodge, Daly, Huyton \& Sanders, 2012; Walsh, 2011). In contemporary Westernized societies such as Aotearoa New Zealand, many different understandings of wellbeing circulate and shape individuals' experiences. Among these understandings is recognition that multiple dimensions including physical, emotional, psychological, spiritual and social dimensions contribute to holistic wellbeing. However, it may be difficult for many individuals to find time and appropriate opportunities to nurture their wellbeing holistically in the current society. Particularly, women who are employed as well as caring for children and elderly people, may lack resources to care for their own wellbeing. Previous research, nevertheless, demonstrates that physical activity such as dancing may offer holistic experiences of wellbeing (Batson \& Wilson, 2014; Goodill, 2005; Karkou, Oliver \& Lycouris, 2017; Murcia, Kreutz, Clift \& Bongard, 2010). The focus of this article is to discuss women's embodied experiences in intergenerational, somatic dance classes, designed using a constructivist feminist perspective, to promote holistic wellbeing in local community.

To provide background for our discussion of empirical findings, we firstly consider multiple understandings of wellbeing, drawing on feminist approaches and contextualising these understandings within our Westernised and neoliberal socio-cultural context. We then draw on relevant research in dance and wellbeing and outline the movement approach offered in somatic dance classes. Within a constructivist theoretical framework, we use a critical feminist ethnographic methodology incorporating in-depth interviews, observations and autoethnographic vignettes 
to gather empirical material. In this article we offer a discussion of three themes relating to embodied experiences of wellbeing arising from this research.

\section{Women's wellbeing, feminism and dance}

The term wellbeing has become commonplace in contemporary Westernized societies and it is associated with varied models, understandings and practices. Although generally understood as a multidimensional conceptualisation of health, contested definitions of wellbeing circulate within the wider socio-cultural context of Aotearoa New Zealand (Durie, 1998; Hone, Jarden \& Schofield, 2014; Mental Health Foundation of New Zealand, 2011; Payne, 2017). While there is no clear consensus on any particular definition of wellbeing (Dodge, Daly, Huyton \& Sanders, 2012), the term has been enthusiastically adopted within popular culture, the fitness and leisure industries, government policy and academic research. In the context of academic research, wellbeing has often been measured using a range of factors that are structural, social and cultural, spiritual, gendered, psychological and physiological, with a noticeable shift over the last twenty years emphasising individualised subjective wellbeing measures (McLeod \& Wright, 2016). In relation to this, the New Zealand Coalition Government has recently introduced The Wellbeing Budget. In this government document, "Wellbeing is when people are able to lead fulfilling lives with purpose, balance and meaning to them" (New Zealand Government, 2019, p.5). While social, cultural and structural factors are addressed as dimensions of wellbeing, individuals are responsible for filling their lives with purpose, balance and meaning. This shift may be considered part of "wider social processes of individualization, reflecting the imbrication of neoliberalism and therapeutic culture" (McLeod \& Wright, 2016, p.5). In this sense, wellbeing has been positioned as a desirable and normalized part of neoliberal, self-improvement projects (Fullagar, 2003). We acknowledge that the ways wellbeing is defined and territorialized in policy, industry, academic research and popular culture all influence women's embodied experiences. Yet we also acknowledge that the Wellbeing Budget (New Zealand Government, 2019) may begin to address the individualised wellbeing towards greater social-cultural responsibility for community as well as individual wellbeing.

We argue that feminist perspectives on wellbeing are particularly important to examine given the complex and often constraining socio-material and economic conditions faced by many women. According to the Ministry of Health (2016), adult women are generally less physically active than men, which has implications for physical and mental health (Sport New Zealand, 2015). Further, with women fulfilling multiple roles working and caring for children, elderly parents and fulfilling community responsibilities, they are facing increasing pressure on both time and energy. As a consequence, women may be more likely to experience stress, anxiety and sleep disruption, as well as ongoing physical and emotional changes across the lifespan (Sánchez-López, Cuellar-Flores \& Dresch, 2012). Given that much of the New Zealand population lives in increasingly urban, culturally diverse, aging and technology-focused communities, women may not have family or social support to assist them in fulfilling multiple roles (Cook, 2007; Kahu \& Morgan, 2008; Spoonley \& Bedford, 2012). Nevertheless, the wellbeing of women is especially important given their central role in family health and wellbeing (Currie, 2004; McIntyre \& Rhodes, 2009), as well as workplace productivity and community development.

Considering the aforementioned issues facing women and the complex debates surrounding wellbeing, there is a compelling need for research within appropriate health and wellbeing activities for women. The research presented within this article moves the discussion beyond the narrowly defined understanding of subjective wellbeing and toward an understanding of what wellbeing can do (McLeod \& Wright, 2016). Despite many circulating and contested definitions of wellbeing, as feminist constructivist researchers we were curious to understand more about women's experiences of wellbeing through embodied practices. We have found it valuable to understand wellbeing broadly as an ongoing experience in which each person embodies a dynamic balance between their resources and the challenges they face: wellbeing is a "dynamic 
dance" (Dodge et al., 2012). As Keogh and Davis (2017) describe, "when we are well, we have a sense of vibrancy and aliveness rooted in our tissues" (p.539). It is from this broad understanding of wellbeing as a dynamic and embodied experience that we engaged in this research. We began our research by attending to women's experiences in local somatic dance classes, to understand if and how participation in community somatic dance might offer experiences of wellbeing. We were curious to explore whether such experiences might extend and potentially push against circulating understandings of wellbeing within this neoliberal context and move toward more holistic wellbeing.

Within the growing body of research literature on wellbeing and dance, it is significant that dance is recognized as a multidimensional activity that offers social as well as emotional, physical, psychological and spiritual benefits (Batson \& Wilson, 2014; Bond, 2019; Goodill, 2005; Karkou, Oliver \& Lycours, 2017; Murcia et al., 2010). Research has suggested that participating in dancing may reduce stress and anxiety, combat depression, challenge participants intellectually, and broaden experiences to support health and wellbeing in working and family lives (Harris, Wilks \& Stewart, 2012; Karkou, Oliver \& Lycouris, 2017). In their research, Murcia, Kreutz, Clift and Bongard (2010) concluded that:

...dancing is perceived to be a multidimensional activity that contributes positively to several aspects of human wellbeing...besides emotional and physical benefits, participants reported dancing to have positive influences on self-esteem, social relations and spirituality as well as providing a significant coping strategy for daily stress and difficult times. (p.159)

However, there is a tendency to locate wellbeing as a self-improvement or preventative strategy for dancers to cope with the demands of training or the profession (such as maintaining self-esteem, developing mindfulness, or managing stress and injury) (Cardinal, 2014; Karkou, Oliver \& Lycouris, 2017; Murcia et al., 2010). Further, research focuses on dance interventions aiming at improving a single aspect of wellbeing within a specific population, and may draw on biomedical approaches that are still common within wellbeing research (Batson \& Wilson, 2014; Karkou et al., 2017; Molloy, Keogh, Krampe \& Guzmán, et al., 2015; Schroeder, Ratcliffe, Perez, Earley, Bowman \& Lipman, 2017).

We note that somatic dance approaches have been identified as offering potential to support wellbeing (Barbour, 2016; Cardinal, 2014; Olsen, 2017), and as such, present a specific context for us to undertake research to investigate women's experiences. In this article, we discuss somatic dance classes called Dancing into Wellbeing that have been offered free within our community for over two years. These classes draw on Land to water yoga from Shin Somatics ${ }^{\circledR}$ (Fraleigh, 2009, 2015) as will be discussed in the following section. Together we researchers are the class facilitator (Barbour) and participants within this community (Clark and Jeffrey). In subsequent sections, we detail our perspectives as critical feminist ethnographers undertaking this research,

\section{Somatic Dance Classes}

Somatic dance classes integrate a range of movement activities that support participants to experience embodied growth and change, and also to recognise the forces that shape us as we live in the world (Burnidge, 2012; Schaeffer, 2015). According to the International Somatic Movement Education and Therapy Association (ISMETA), participating in somatic practices supports participants to "experience an embodied sense of vitality and create both meaning for and enjoyment of life" (ISMETA, 2019, para. 3). Recent international research provides evidence of the value of somatic dance practices for wellbeing (Batson \& Wilson, 2014; Bond, 2019; Fraleigh, 2015; Olsen, 2017; Weber, 2019). For example, Olsen comments that in somatic dance "we recover the easeful coordination in the nervous system that underlies creative thinking and healthful imagination - cultivating wellness in relation to self, other, and the natural world" (2018, p. 191). While research linking dance and wellbeing (Karkou et al., 2017), and dance and quality of life (Bond, 2019) is growing, few studies focus specifically on somatic dance and holistic wellbeing, or provide empirical material from participants revealing their embodied experiences. 
In Aotearoa New Zealand, research into somatic dance has been limited to date. In previous research by Barbour (2014), dancers expressed that dancing itself had the potential to offer opportunities to enhance wellbeing, particularly when integrated with yoga and somatic practices. Further work with older adults suggests that "the performance of somatic-based dance movements, even if only practiced once a week, may produce wellbeing benefits for older adults and an associated impact on their increased capability in habitual physical activities" (Molloy et al., 2015, pp.177-178). Our research engages participants in somatic intergenerational dance classes and gathers their reflections on holistic wellbeing.

The Dancing into Wellbeing classes offered in our local community include the processes of Land to water yoga from Shin Somatics ${ }^{\circledR}$ (developed by Sondra Fraleigh $(2009,2015)$ and affiliated to ISMETA). The ethical principles that guide these particular classes include enhancing somatic awareness, engaging in movement as a wellbeing practice, supporting agency and freedom, and creating caring and belonging (Barbour, 2016).

A typical Dancing into Wellbeing class engages participants in everyday movement patterns beginning with walking upright on land, descending to hands and feet, crawling, sitting and lying as a practice of retracing "our personal human development back to its watery soma source" (Fraleigh, 2009, p.xiii). Following rest on the floor and breathing activities, the class returns participants to standing through developmental yoga explorations, culminating in open improvisation (for more detailed discussion see Barbour, 2016; Fraleigh, 2009; Schaeffer, 2015). It is important to emphasize that this class offers movement patterns using a method that encourages embodied awareness, individual creativity, agency and reflection. This approach should be understood as quite distinct from demonstration and instructional approaches designed to teach a codified exercise which should be repetitively and correctly executed, as commonly undertaken in dance technique or physical exercise classes (Markula, 2011). Instead, Fraleigh encourages facilitators of Shin Somatics ${ }^{\circledR}$ to develop pedagogical nuances and creativity to respond to the needs of different communities.

\section{Methodology}

\section{Critical Feminist Ethnography}

Our qualitative research is located within constructivism and critical feminism, and assumes that knowledge is created through constructed and embodied ways of knowing, within particular socio-cultural and political contexts (Barbour, 2011; Guba \& Lincoln, 1989; Hesse-Biber \& Leavy, 2007; Markula \& Silk, 2011). Therefore, we understand our roles as feminist constructivist researchers working together with participants as active co-constructors of knowledge within shared embodied practices. Researching in the field of somatic dance classes, we, as authors, bring familiarity with a range of dance, somatic and yoga practices in community, institutional and workplace settings. Importantly, we acknowledge that our interactions with the participants shape the meaning-making processes and embodied knowledges that emerge from this research. We do not attempt to hide our role in the interpretation and analysis of empirical material. Rather, we view ourselves as working closely with our participants, the empirical material collected, and the context in which this research is conducted (Denzin \& Lincoln, 2005).

We utilise our insider observations and reflexivity as participants within the dance community to gain deeper insights into these shared experiences. Feminist ethnography (Beal, 2018; Madison, 2012) provides a methodology for investigating the embodied lived experiences of women and examining the ways in which local and personal embodied experiences are shaped by wider political and socio-cultural contexts. Madison (2012) argues that a concern about unfairness and injustice creates a "sense of duty and commitment based on principles of human freedom and well-being" that drives critical ethnographic research (p. 5). From our perspectives, in order to voice women's wellbeing experiences and to potentially expand our understandings of wellbeing within the individualising, illness/injury prevention, selfimprovement projects and workplace productivity agendas circulating in this neoliberal 
socio-cultural context, we need to participate, observe and listen to women's stories. Thus, our intention in this feminist ethnography is to support a variety of voices, sharing embodied personal and social experiences within wellbeing practices that may enhance understandings.

\section{Participants}

Women who participated regularly in the free open Dancing into Wellbeing classes offered on Friday afternoons were invited to participate in the research. More than 50 women attended over two years, bringing a range of experiences as dancers, non-dancers, health professionals and yoga teachers. Participants came from a variety of cultural backgrounds, including Pākehā (non-indigenous New Zealanders), indigenous Māori women, recent immigrants and international students from China, United Kingdom, Canada, Malaysia, the Pacific Islands and Europe. They ranged in age from 18 to 75. Eight regular attendees agreed and were available to participate in an interview. In addition, as participating researchers we were also members of the community. Consequently, the research reflects the experiences of 11 women 1870 years old, Pākehā or recent immigrants from Canada and England, and who were committed attendees. We note that the research does not fully represent the diversity of the women who participated in the classes. This is a limitation we identify within this initial research that offers potential for future inquiry. Pseudonyms were allocated to the participants to retain their anonymity.

\section{Collection and Analysis of Empirical Material}

In this project we engaged as critical feminist ethnographers in order to prioritize women's voices and embodied experiences (Beal, 2018; Hesse-Biber \& Leavy, 2007; Rigg, Cook \& Murphy, 2014). We aimed to create space to listen to the embodied lived experiences of the intergenerational group of women through in-depth interviews with regular participants. In-depth interviews (Hesse-Biber \& Leavy, 2007) with eight women were conducted over two months by Marianne Clark, using an interview guide structured according to the topics of wellbeing, dance and movement class experiences and the relationship between wellbeing and class participation. The interviewees were familiar with Clark as she had participated in some classes alongside them. This shared experience allowed rapport to be developed easily and conversational, at times intimate, dialogue to unfold.

Guided by the thematic analysis process outlined by Braun and Clarke (2006), Allison Jeffrey initially identified a number of common themes during the process of transcribing interviews. Together, we three researchers then collectively engaged with the transcripts, taking care to identify interesting unique insights, and utilize the women's own words in identifying common themes. The interview material revealed broad themes that we grouped as relating to: expanded understandings of wellbeing through movement; movement for movement's sake; and the value of intergenerational dance classes.

We also document some of our personal, embodied experiences in the somatic dance classes through autoethnographic writing. Autoethnography refers to writing (graphy) back and forth between self (auto) and culture (ethno). It is an academic genre of writing that moves beyond autobiography to connect the researcher's personal experience to political, cultural and social understandings (Barbour, 2011; Ellis, 2004; Humberstone \& Cutler-Riddick, 2014). Having participated in the Dancing into Wellbeing classes ourselves, we shared our own experiences together, noting when our experiences aligned with the broad themes arising from the interviews and identifying critical moments of resonance or incongruity. Through sharing experiences, reflections and notes, we identified a critical moment relating to each of the broad themes and wrote an autoethnographic vignette. These vignettes were collectively edited to encompass both the facilitator's pedagogical notes (most evident in the Theme 1 vignette) and our participant observations. 
Our themes and insights about wellbeing were then brought into dialogue with selections from the range of sociocultural understandings on women's wellbeing circulating in contemporary Aotearoa New Zealand. Throughout the following sections, we share and discuss these themes and insights, remaining cognizant of the research in dance and wellbeing and also seeking to highlight unanticipated insights using the women's words. The vignettes function to narratively frame each theme and bring the embodied experience of Dancing into Wellbeing to life on the page.

\section{Findings}

\section{Theme 1: Expanding understandings of wellbeing through movement}

Entering the studio, I remove my shoes and socks and wriggle my toes, bare feet connecting with the smooth floor; a small sense of relief as my soles feel wood, air and freedom. Alongside me are other women, some older, some younger, some familiar, and some new. As we greet each other in this lovely studio, I feel a shared sense of reverence for our time and place to move.

Class starts with gentle encouragement and we begin to walk slowly and purposefully around the room, inhabiting the space, breathing and bringing attention into our bodies. We are guided into the opening exercise, as facilitator, Karen's voice is gentle, soothing:

'In walking, observe your pace, without judgment.

Allow your pace to slow until you find yourself pausing, one foot in front.

Try a little, gentle rocking back and forward, shifting your weight easily.

Rocking forward allow the heel of the back foot to rise off.

Rocking back allow the front toes to lift.

As you rock forward, add an easy opening through sternum, opening your heart.

And as you rock back allow an easy fold in the back knee and hips.

Now allow your eyes to open out to your own horizon while rocking forward, opening your heart.'

Rocking, shifting, opening, folding. New connections are created and others let go. Heel, sternum, heart, eyes. Bodily possibilities are expanded. All of these movements come together and my body delights in lingering in new kinesthetic horizons.

Women entered the studio for Dancing into Wellbeing classes with varied movement experiences, life circumstances and reasons for attending. When we asked the women how they understood wellbeing, they referred to some of the many circulating definitions of wellbeing, and they extended these understandings. In describing wellbeing they spoke of a harmony or balance between different aspects or dimensions. These dimensions were described using words typically referenced within wellbeing literature and included emotional, physical, mental, social, health and spiritual dimensions. Their definitions also encompassed a broader understanding of wellbeing incorporating dimensions of soul, connection with others and nature, moving, activity, creativity, safety, resting, feeling at peace and feeling good. For example:

Rainbow: '[wellbeing] is a sense of balance between all of the different aspects of your life...the emotional, physical, mental elements working together.'

Groovy: 'wellbeing is tied into connection...we make connections with ourselves and other people and with nature.'

Jay: '[wellbeing is about] having contact with friends and family, music'

Rainbow: 'if you have good relationships with other people you have a good sense of wellbeing.' 
While the first comment aligns with understandings of wellbeing as managing different aspects or dimensions, such as balancing mental, physical and social dimensions of wellbeing, the women's comments also emphasised the importance of the relationships between one's self and others, communities and nature. Thus, circulating perspectives of health that focus singularly on the state of the physical body or on mental and emotional health, were not the main focus of the women's comments. Our observations and the interviews (as demonstrated above) revealed that the participants embraced the class as a meaningful way to engage in multiple dimensions of wellbeing.

For some women, the somatic dance classes provided a safe opportunity to move with wellbeing issues that were causing them discomfort and/or stress. Entering the class with a diminished sense of wellbeing, these women viewed the movements as facilitating a sense of relaxation. These same women identified this as valuable for their lives more broadly and beyond the studio.

Lee: '[the class allows me to] relax and de-stress and set aside all of the anxieties...after I leave I find that I am way less stressed than when I came in.'

Dove: 'When I come out of the class - my soul is just so happy and so that blows through to just my mind being happy and my body feeling happy and then I kind of just have that nice feeling of being calm and centered.'

The sense of calm enabled by the movement experiences in class further contributed to women's wellbeing by enhancing their ability to fulfill their roles in their professional, familial and social lives in meaningful ways. It seemed that some women gained understanding of how moving is related to wellbeing and how it can contribute to one's community and family life (Payne, 2017). After the classes several of the women describe feeling better equipped to navigate difficult relationships and work-life balance.

Lee: 'When I left the difference in how I felt was amazing. I left feeling as though I had just been given this charge of energy and probably what I should have done is gone home and slept and capitalized on [it]. But I was like oh yeah I can work for another two days now. That sense of wellbeing is what keeps me coming back. Maybe it is time out of a busy schedule and it's hard to prioritize that sort of time but knowing that it will actually benefit me and make me more productive and make me feel better is amazing.'

This comment illustrates the important role that somatic dance movement may play in women's lives in supporting wellbeing. While Lee commented that the Dancing into Wellbeing classes offered her the potential for further productivity at work, she also relished that it was the feeling of energy and wellbeing that kept her coming back. The value of the experience for her reached beyond neoliberal agendas of individual responsibility for workplace productivity.

These findings also expand and challenge circulating neoliberal discourses that situate physical activity and exercise as primarily ways to alleviate health risks (Dworkin \& Wachs, 2009; Fullagar, 2012). While a physical aspect of wellbeing was important to these women, instead of understanding the body simply as ill, in pain or healthy, the body was one aspect of the shifting, continuously evolving experience of wellbeing. The meanings these women make of and ascribe to these movement practices provide alternative and more expansive experiences than afforded by dominant health promotion that constructs physical activity as a means to achieve a fixed health status to contribute to productivity and/or a disciplined, desirable body (Ayo, 2012; Markula, 2011). Instead, women's stories illustrate how participating in movement practices enabled meaningful relationships, connections and harmony (Clark, 2017; Clark \& Markula, 2017).

In this sense, we argue that there is potential to reclaim wellbeing from the clutches of neoliberalism as expressed through individualism in the fitness industry and popular culture. Instead we suggest an alternate view of wellbeing as experiences that support personal and social change through continually shifting, balancing and evolving relationships, connections and harmony. In this way, we push back against dominant understandings of wellbeing, suggesting that wellbeing is not something to be achieved through ticking boxes on a scale. Rather, it may be more helpful to view wellbeing as a continual process of inquiry with no single outcome. 


\section{Theme 2: Movement for Movement's Sake}

In this moment I feel my body touching the cool floor beneath me, allowing experiencing weightiness. Feeling myself soften, I am safe in this place (away from the gaze of the world). Savoring my contact with the floor, allowing my entire experience to absorb me, I integrate cues from our teacher. The cadence of the music, the feel of breath flowing through my body and the glimpses of other dancers expressing through shared movement patterns all infuse this moment. The others in the studio are moving as they need to, but (unlike the outside world) I am not checking in to make sure I am moving appropriately. Instead I'm deciding I want to remain still for a while. Lying on the floor I let out a sigh of relief allowing myself to drift into a deep relaxation. The pressures of productivity ooze from my body. There is nothing I need to be doing in this moment. Closing my eyes, the muscles of my face softening, I'm smiling. I begin rolling gently from side to side, without agenda. My movements are not being graded or scrutinized and in this cloak of acceptance I am moving to my own rhythm, following my own interests as they arise in this moment.

Importantly, for many women the Dancing into Wellbeing classes provided opportunity to move in expressive, creative ways. A marked difference that the women noted in these classes (as compared to more structured dance disciplines like ballet), was the ability to move without the added pressure of an external outcome such as correct execution of a skill or performance for the approval of others. Expression encouraged within these classes prompt new and different experiences of and relationships to their own bodies and others around them, thus enhancing their wellbeing. Drawing on the voices of our participants, we describe this as movement for movement's sake.

Star: 'You don't feel as though you are doing the movement to achieve the perfection of that movement. It's kind of just move to enjoy, move to feel well, move to move.'

Cece: 'This class is so beneficial because it is less structured... Doing the class in this way makes you ponder what is important. Other classes place importance on questions like "what is the end product here" but it's like "NO that's not the point" it's the actual doing of it that is the important thing.'

The women also found value in being able to move without fear of having their movements labeled as right or wrong (Correia, Rosado, Serpa \& Ferreira, 2017; emerald \& Barbour, 2015). While some research on wellbeing includes accomplishment as one precursor for wellbeing (Seligman, 2011), it may be equally important to provide women the opportunity to freely express themselves outside of potentially stressful expectations and judgements.

Cece: 'There's no judgement... it's not about getting the movements right, it's about feeling the process internally.'

Lee: 'I knew that the movement classes were framed in terms of moving as you are able ... it is really good to know that you are not going to be expected to match or replicate. You can try things out and then if it doesn't work no one is going to be looking at you ... you are not going to feel like you have failed.'

These ideas surrounding wellbeing as something that is without agenda move beyond current understandings of wellbeing as checklists (e.g., Hone, Jarden \& Schofield, 2014). As suggested by the women, it may be more suitable to consider the fluidity of wellbeing as an experience that is felt and offers freedom in moving.

While moving freely without agenda offered the women the opportunity to enjoy movement away from daily pressures, the particular sequence of the classes helped the women to explore and expand their repertoire of movement experiences throughout their day. Freely moving in unconventional ways opened the women up to moments of wellbeing that they would not encounter throughout typical work and home life. In particular, the resting and breathing activities (Fraleigh, 2009, 2015) allowed the women the opportunity to feel connected to the floor beneath them and to explore a different relationship to gravity.

Rainbow: 'I really like the floor stuff ... I enjoy being on the ground and rolling around and not being this bipedal humanoid thing.' 
Star: 'I like being on the floor which sounds probably a bit strange but I just always like being on the floor ... it's just sort of that relationship with the floor ... it is kind of like I am working with the floor.'

Women's relationship with the floor allowed them to access an expanded repertoire of movement experiences, being less bipedal and upright, and more grounded. This active engagement and creation of new movement experiences is similarly reflected in their re-imaginings of wellbeing, which may be seen to contrast dominant societal norms for women that require appropriate and carefully controlled feminine movement behaviour (Perloff, 2014; Pottinger, 2017).

Lee: 'This class gives you the opportunity to do movements without inhibition ... you sort of forget how to do that and that is a nice space to make for yourself ... the way life is these days it is really hard to make a space to be that person.'

Today, women face a growing number of family roles and heightened societal pressure to cope emotionally and appear resilient amongst unrealistic expectations both at work and at home (Mountz et al., 2015). These mounting daily stressors are often compounded by images on social media depicting women effortlessly achieving insurmountable tasks with apparent ease. Amongst the pressure assaulting their daily lives, women are expected to take charge of their wellbeing within a society that encourages neoliberal ideals of self-improvement (Nash, 2018; Thorpe, Toffoletti \& Bruce, 2017). In contrast, these classes allowed the women the chance to unwind and relax in ways they actively found meaningful and were not prescribed as rehabilitation, exercise or therapy.

This opportunity to move for movement's sake is particularly important as most actions taken within our neoliberal world are done with the expectation of a desirable outcome or gain (Genz, 2017). This expectation to achieve and the practice of comparing oneself against a set of measurable attributes can be seen even within the policy and practices of wellbeing (McLeod \& Wright, 2016). Further, for women in a movement context, often skill acquisition or health goals (e.g., weight loss or rehabilitation) are the accepted rationales for pursuing any physical activity, including dance (emerald \& Barbour, 2015). On the contrary, these classes did not offer a fashionable self-improvement project, a rehabilitation program nor a targeted intervention. Instead, the classes were free to all those who were interested, attracting a varied and intergenerational group.

\section{Theme 3: Intergenerational community dance}

Moving together, I feel the energy in the studio shifting around me, the pace and intensity of movement slowly increasing. In my peripheral vision I catch a trace of movement from Rainbow and following her, I commit myself to her youthful vibrancy, rolling onto my side, sweeping my hand across the floor, spiraling spine, soles and palms connecting with the floor under me, pelvis shifting over my feet, spine aligning while unfolding, away traveling through the space. Catching glimpses of women moving, swirling momentum, paths brushing mine like eddying currents in a stream. Alongside Groovy, my tempo adapts, becoming more rhythmic as the swing of her arms connects into the swing of the music. Feeling my smile widen, reflecting hers, the years between us disappear as we both share this playful movement quality, dancing together before the currents pull us in other directions. And then I'm rolling again, nourishment in moving with others, embodying wellbeing, simply sharing a commitment to being together.

These Dancing into Wellbeing classes offered an opportunity in which women moved together in a community context that was intended to be accessible for most bodies, cultivated embodied awareness, used inclusive pedagogy and invited creative expression (Amans, 2008; Barbour, 2016; Fraleigh, 2015). Including an improvisational section near the end of the class allowed women to follow their own interests, engaging deliberately or subtly with others as they used their embodied ways of knowing to enhance wellbeing (Barbour, 2011). This improvisation supported agency in identifying movement that was personally relevant from the class (or elsewhere), and freedom in moving without a specific outcome (Barbour, 2016). Additionally, the vignette above suggests that there is potential for a deeper understanding of the ways in which such classes might foster social and community experiences, extending understandings of the benefits for women 
within mixed ages and intergenerational groups. The women interviewed expressed an appreciation of the intergenerational context:

Cece: 'It's nice to sort of have a mixture of ages, to find other older women... another opportunity to move but in your own way and I've always liked to have a mix of ages in all the classes because there is a mix of energy being in with the younger people... everybody is accepted for who they are and how they move.'

Working alongside women of varying ages prompted some dancers to identify and question the potential for ageism in dance, particularly in Westernized studio-based dance that targets younger people and thus, often excludes aging bodies (Bolwell, 2017; emerald \& Barbour, 2015; Humberstone \& Cutler-Riddick, 2014).

Star: 'I feel like dance can be quite ageist in some ways...it'll only be for young and able, or dance for older women. But why don't we all do it together... that's really nice.'

Melody: 'When we were talking about being within the class, being with [different] ages, what was also lovely was being with...very skilled dancers, practiced, disciplined dancers, who I have watched dance on a public stage and yet feeling that it was absolutely fine that I was dancing with them...Here we have people who are not only living at different stages but with greatly different experiences in dance.'

Women saw the benefit in imagining how they might move differently through seeing dancers of other ages. Experiences of moving together supports awareness, curiosity and acceptance of different ways of moving including dancing with an ageing body; experiences likely to be undervalued because they do not translate into achievement and outcomes. There is learning in such lived experiences that potentially offers insight into other ways of being.

Lee: 'I'm interested in how some of the older women feel about all of this just because if you can look forward a bit into the future...sometimes you can do things which are better...I like the fact that the women of my mom's age are coming along and enjoying it and I like the fact that the younger ones come too.'

Rainbow: 'I really do like the element of moving with a whole range of people who are different ages. And I don't really know quite how to explain it all and why, but I think it's just really cool to be in a room and you see that there is someone who is 50 and there is someone who is 17 and it's kind of nice'

While Rainbow did not comment on ageism in dance (Bolwell, 2017; Humberstone \& Cutler-Riddick, 2014), her comments reveal an appreciation that there are other benefits in dancing with women of different ages that have more to do with social values (Barbour, 2018). Groovy, drew on themes of social connection and freedom from expectation, concluding that moving together within this setting supports her wellbeing.

Groovy: 'It ties into the idea of community for me because it is...harder than you might think for people to connect... joining things and being part of [these classes] can lead to social connections with other people...I think wellbeing is tied into it... I think we make connections with ourselves and with other people and with nature and whatever it is we believe in and that's where wellbeing comes in from my perspective.'

Through the improvisational and social aspects of the Dancing into Wellbeing classes, women integrated somatic and other forms of knowledge and experienced agency in making movement choices. They felt freedom in moving without judgement and connecting with women of different ages. In the moment of moving, of embodied ways of knowing, women reconciled this knowledge in personally meaningful ways, in relationship with others and the world as part of social and community life (Barbour, 2016).

Research identifies that dance may support wellbeing across the lifespan, as revealed in a range of studies with different age groups including children, young women and elderly populations (Schroeder et al., 2017). Even though studies have revealed the benefits of dance for all ages, limited research has considered the impact of intergenerational dance classes. The women in this research identified that dancing within an intergenerational group further supported their wellbeing through fostering connections with other women simply through moving together (without 
necessarily having to talk), providing opportunities to learn from the wisdom of more mature dancers and gaining inspiration from younger, energetic dancers.

\section{Discussion}

Our research focused on women's experiences of an integrative somatic dance approach and the potential to reveal an expansive understanding of wellbeing (Barbour, 2016; Cardinal, 2014; Merchant, 2017; O’Brien, 2016). In Aotearoa New Zealand, dance and yoga/Pilates are common forms of activity chosen by women to support their wellbeing (Sport New Zealand, 2015). However, there is limited research into somatic dance and wellbeing, particularly that focuses on women's embodied experiences (Merchant, 2017; Murcia, Kreutz, Clift \& Bongard, 2010). Much international research uses brief intervention studies locating a single physical or psychological focus within a specific practice such as yoga or aerobics. The dance and wellbeing research offers valuable insights, although again is limited in specifically addressing somatic dance and wellbeing (Bond, 2019; Karkou, Oliver \& Lycouris, 2017).

Our interviews revealed that women of all ages have complex experiences of wellbeing, affected by changing relationships to their bodies, and demanding schedules balancing family, community and working lives. Consequently, these women articulated a need for movement practices that foster their wellbeing. While such movement practices may not be beneficial or enjoyable for all, our small study suggests that there are multiple benefits in movement practices that allow women of varying ages to connect with their bodies and in a non-judgmental environment.

Both the social environment and the somatic dance content in this class allowed women to cultivate new connections and relationships with others, and to actively create their own understandings of what wellbeing means to them in their daily lives. Women actively reflected on wellbeing in their lives, not as a state to achieve or reach, but as a continuous fluctuating experience. In this sense, Dancing into Wellbeing classes:

refuse the dominant logic of health as an outcome of exercise and instead value a relation to the body based on a ethics of attentiveness, of affectivity or care, and even of pleasure and excess that is beyond a calculative logic. (Fullagar, 2003, p.57)

This study, thus, expands upon our understandings of wellbeing in Aotearoa New Zealand and has implications when working with any model for wellbeing. Aligned with the women in this study, it is helpful to understand wellbeing as a holistic experience - a dynamic dance - rather than a balanced state across a fixed set of dimensions.

The Dancing into Wellbeing classes allowed both for the enhancement of wellbeing and for women's active engagement in reflection and cultivation of a sense of caring for one's self and others that was responsive to context and circumstance, not defined externally by policy or media. Participants referenced dominant discourses of health that encourage exercise and movement, but also reflected on the impact of their lived experiences that is often absent in related literature. Additionally, the women revealed the value of sharing movement experiences with women of different ages, an unexplored potential of somatic dance and movement practices that both appeal to and are accessible to women across the lifespan.

Consequently, we conclude that our research participants found the Dancing into Wellbeing somatic dance classes valuable as they cultivated a wide range of accessible movement experiences for women of all ages in order to encourage active engagement and embodied ways of knowing. Somatic dance classes that foster women's ability to reimagine wellbeing in their own terms and provide meaningful movement experiences are important in order to expand and disrupt neoliberal understandings of physical activity, health and wellbeing. Further, such classes support women's sense of connectedness and ability to function well in their familial, social and community contexts. 


\section{Acknowledgements}

Thanks to all of the participants in Dancing into Wellbeing and Movement for Wellbeing classes in New Zealand; to Sondra Fraleigh and the Eastwest Institute for Yoga, Dance and Movement Studies; and to The School of Arts, The University of Waikato.

\section{References}

Amans, D. (2008). An introduction to community dance practice. Basingstoke: Palgrave Macmillan.

Ayo, N. (2012). Understanding health promotion in a neoliberal climate and the making of health conscious citizens.

Critical Public Health, 22(1), 99-105.

Barbour, K.N. (2011). Dancing across the page: Narrative and embodied ways of knowing. Bristol, UK: Intellect Books.

Barbour, K.N. (2014). Searching for Bliss: Insights and challenges in yoga and contemporary dance choreography. Dance Research Aotearoa, 2, 79-100.

Barbour, K.N. (2016). Embodied values and ethical principles in somatic dance classes: Considering implicit motor learning. Journal of Dance and Somatic Practices, 8(2), 189-204.

Barbour, K.N. (2018). Embodied ways of knowing: Living, writing and dancing. In B. Wheaton, J. Caudwell, L. Mansfield \& R. Watson, (Eds.), The Palgrave Handbook of Feminisms and Sport, Leisure and Physical Education (pp. 209-226). London: Palgrave Macmillan.

Batson, G., \& Wilson, M. (2014). Body and mind in motion. Dance and neuroscience in conversation. Bristol, UK: Intellect Books.

Beal, B. (2018). Feminist ethnographies in sport and leisure. In The Palgrave Handbook of Feminism and Sport, Leisure and Physical Education (pp. 227-242). Palgrave Macmillan, London.

Bond, K. (Ed.). (2019). Dance and the quality of life. Cham, Switzerland: Springer

Bolwell, J. (2017). Wellbeing and the aging dancer. In V. Karkou, S. Oliver, \& S. Lycouras (Eds.), The Oxford handbook of dance and wellbeing (pp. 311-328). Oxford, UK: Oxford University Press.

Braun, V., \& Clarke, V. (2006). Using thematic analysis in psychology. Qualitative Research in Psychology, 3(2), 77-101. Burnidge, A. (2012). Somatics in the dance studio: Embodying feminist/democratic pedagogy. Journal of Dance Education, 12(2), 37-47.

Cardinal, M.K. (2014). SPICE'S: Wellness dimensions applied to dance with advice for teachers. Journal of Physical Education, Recreation \& Dance, 85(3), 3-7.

Clark, M. I. (2017). Multiple bodies: In the ballet studio with adolescent ballet dancers. In Markula, P., \& Clark, M.I. (Eds.) The Evolving Feminine Ballet Body, (pp. 49-77). University of Alberta.

Clark, M. I. \& Markula, P. (2017). Foucault at the barre and other surprises: A case study of discipline and docility in the ballet studio. Qualitative Research in Sport, Exercise, \& Health, 9, 1-18.

Cook, R. (2007). New Zealand families. Marriage and Family Review, 41(3), 241-259.

Correia, M., Rosado, A., Serpa, S., \& Ferreira, V. (2017). Fear of failure in athletes: Gender, age and type of sport differences. Revista iberoamericana de psicología del ejercicio y el deporte, 12(2), 185-193.

Currie, J. (2004). Motherhood, stress and the exercise experience: Freedom or constraint? Leisure Studies, 23 (3), $225-242$.

Denzin, N. K., \& Lincoln, Y. S. (2005). The Sage Handbook of Qualitative Research (3rd ed.). Thousand Oaks, USA: Sage Publications Ltd.

Dodge, R., Daly, A.P., Huyton, J., \& Sanders, L.D. (2012). The challenge of defining wellbeing. International Journal of Wellbeing, 2(3), 222-235.

Durie, M. (1998). Whaiora: Māori health development (2nd ed.). Auckland, New Zealand: Oxford Uni Press. 
Dworkin, S. L., \& Wachs, F. L. (2009). Body panic: Gender, health, and the Selling of Fitness. New York, USA: NYU Press.

Ellis, C. (2004). The ethnographic I: A methodological novel about autoethnography. Walnut Press, CA: Altamira Press. emerald, e., \& Barbour, K.N. (2015). 'I'll go back next week - it's complicated': Returning to dance with the help of Bourdieu. In lisahunter, W. Smith, \& e. emerald (Eds.), Pierre Bourdieu and physical culture (pp.27-36). NY: Routledge.

Fraleigh, S. (2009). Land to water yoga: Shin somatics moving way. Bloomington, IN: iUniverse.

Fraleigh, S. (Ed.). (2015). Moving consciously: Somatic transformations through dance, yoga and touch. Chicago, IL: University of Illinois Press.

Fullagar, S. (2003). Governing women's active leisure: The gendered effects of calculative rationalities within Australian health policy. Critical Public Health, 13(1), 47-60.

Fullagar, S. (2012). Governing healthy family lifestyles through discourses of risk and responsibility. In J. Wright \& V. Harwood, (Eds.), Biopolitics and the 'Obesity Epidemic. Governing bodies (pp. 116-134). New York \& Oxon, UK: Routledge.

Genz, S. (2017). 'I have work... I am busy... trying to become who I am': Neoliberal girls and recessionary postfeminism. In M. Nash \& I. Whelehan, (Eds.), Reading Lena Dunham's Girls: Feminism, postfeminism, authenticity and gendered performance in contemporary television, (pp. 17-30). Cham: Palgrave Macmillan.

Goodill, S.W. (2005). An introduction to medical dance/movement therapy: Health care in motion. London: Jessica Kingsley Publishers.

Guba, E. G., \& Lincoln, Y. S. (1989). Fourth generation evaluation. Newbury Park, CA: Sage.

Harris, N. Wilks, L. \& Stewart, D. (2012). HYPEd-up: Youth dance culture and health. Arts \& Health, 4(3), $239-248$. DOI: $10.1080 / 17533015.2012 .677849$

Hesse-Biber, S.N., \& Leavy, P.L. (Eds.). (2007). Feminist research practice: A primer. Thousand Oaks, USA: Sage Publications.

Hone, L., Jarden, A., \& Schofield, G. (2014). Psychometric properties of the Flourishing Scale in a New Zealand sample. Social Indicators Research, 119(2), 1031-1045.

Humberstone, B. \& Cutler-Riddick, C. (2014). Older women, embodiment and yoga practice. Ageing and Society, 1-21. doi:10.1017/S0144686X1400018X

International Somatic Movement Education and Therapy Association. (2019). Scope of practice. ISMETA: Growing the field of somatic movement education and therapy. Available: https://ismeta.org/about-ismeta/scope-of-practice

Kahu, E.R., \& Morgan, M. (2008). Making choices: Contradictions and commonalities in the valuing of caring and working by government policy and first time mothers. New Zealand Research in Early Childhood Education, 11, $1-17$.

Karkou, V., Oliver, S., \& Lycouris, S. (Eds.). (2017). The Oxford Handbook of Dance and Wellbeing. New York, NY: Oxford Uni Press.

Keogh, A.F., \& Davis, J. (2017) Cultivating the felt sense of wellbeing. How we know we are well. In Karkou,V., Oliver, S. \& Lycouris, S. (Eds.), The Oxford Handbook of Dance and Wellbeing, (pp.535-546). New York, NY: Oxford Uni Press.

Madison, D.S. (2012). Critical ethnography: Method, ethics and performance. Thousand Oaks, CA: Sage.

Markula, P. (2011). 'Folding': A feminist intervention in mindful fitness. In Kennedy, E., \& Markula, P. (Eds.). Women and Exercise: The Body, Health and Consumerism (Vol. 5). (pp.60-78). New York, NY: Routledge.

Markula, P., \& Silk, M. L. (2011). Qualitative research for physical culture. London, UK: Palgrave Macmillan. 
McIntyre, C.A., \& Rhodes, R.E. (2009). Correlates of leisure-time physical activity during transitions to motherhood. Women \& Health, $49(1), 66-83$.

McLeod, J., \& Wright, K. (2016). What does wellbeing do? An approach to defamiliarize keywords in youth studies. Journal of Youth Studies, 19(6), 776-792.

Mental Health Foundation of New Zealand. (2019). Ways to wellbeing. Auckland, New Zealand: Mental Health Foundation of New Zealand. Available: https://www.mentalhealth.org.nz/home/ways-to-wellbeing-2/

Merchant, S. (2017). Therapeutic movement/leisure practices. In M.L.Silk, D.L. Andrews \& H. Thorpe, (Eds.), Routledge Handbook of Physical Cultural Studies, (pp.72-83). Florence: Taylor \& Francis.

Ministry of Health. (2016). Annual update of key results 2015/16: New Zealand health survey. Wellington, New Zealand: Ministry of Health. Available: https: www.health.govt.nz/publication/annual-update-key-results-2015-16-newzealand-health-survey

Molloy, F., Keogh, J., Krampe, J., \& Guzmán, A. (2015). Dance mobility: A somatic and dance programme for older adults in New Zealand. Body, Movement and Dance in Psychotherapy, 10(3), 169-180.

Mountz, A., Bonds, A., Mansfield, B., Loyd, J., Hyndman, J., Walton-Roberts, M., Basu, R., Whitson, R., Hawkins, R., Hamilton, T. \& Curran, W. (2015). For slow scholarship: A feminist politics of resistance through collective action in the neoliberal university. ACME: An International E-Journal for Critical Geographies, 14(4), 12361259.

Murcia, C.Q., Kreutz, G., Clift, S., \& Bongard, S. (2010). Shall we dance? An exploration of the perceived benefits of dancing on well-being. Arts \& Health, 2(2), 149-163.

Nash, M. (2018). Empowering "Sporty Sisters" through “Active Living”: A feminist multi-modal critical discourse analysis of the Lorna Jane Fitness Fashion website. In K. Toffoletti, H. Thorpe \& J. Francome-Webb, (Eds.), New Sporting Femininities. New Femininities in Digital, Physical and Sporting Cultures, (pp. 277-298). Palgrave Macmillan, Cham.

New Zealand Government. (2019). The Wellbeing Budget. Available: https://treasury.govt.nz/publications/wellbeingbudget/wellbeing-budget-2019

O'Brien, E. (2016). Move2Love and vibrancy: Community dance/fitness. Women \& Therapy, 39(1-2), 171-185.

Payne, H. (2017). The bodymind approach. Supporting the wellbeing of patients with chronic medically unexplained symptoms in primary healthcare in England. In V. Karkou, V., S. Oliver, S., \& S. Lycouris. (Eds.), The Oxford Handbook of dance and wellbeing, (pp.769-788). NY: Oxford Uni Press.

Perloff, R. M. (2014). Social media effects on young women's body image concerns: Theoretical perspectives and an agenda for research. Sex Roles, 71(11-12), 363-377.

Pottinger, L. (2017). Planting the seeds of a quiet activism. Area, 49(2), 215-222.

Rigg, K.K., Cook, H.H., \& Murphy, J.W. (2014). Expanding the scope and relevance of health interventions: Moving beyond clinical trials and behavior change models. International Journal of Qualitative Studies on Health and Well-being, 9(1), 24743.

Sánchez-López, M. D. P., Cuellar-Flores, I., \& Dresch, V. (2012). The impact of gender roles on health. Women \& Health, 52(2), 182-196.

Schaeffer, C.A. (2015). Living Shin. In S. Fraleigh, (Ed.), Moving consciously: Somatic transformations through dance, yoga and touch, (pp. 75-92). Chicago, IL: University of Illinois Press.

Schroeder, K., Ratcliffe, S.J., Perez, A., Earley, D., Bowman, C., Lipman, T.H. (2017). Dance for health: An intergenerational program to increase access to physical activity. Journal of Pediatric Nursing, 37, 29-34. 
Seligman, M. E. (2011). Flourish: A visionary new understanding of happiness and well-being - and how to achieve them. Sydney, Australia: Random House Australia Pty Ltd.

Spoonley, P., \& Bedford, R. (2012). Welcome to our World? Immigration and the reshaping of New Zealand. Auckland: Dunmore Publishing.

Sport New Zealand. (2015). Sport and recreation in the lives of New Zealand adults. Active New Zealand Survey Results (2013/14). Wellington: Sport New Zealand.

Thorpe, H., Toffoletti, K., \& Bruce, T. (2017). Sportswomen and social media: Bringing third-wave feminism, postfeminism, and neoliberal feminism into conversation. Journal of Sport and Social Issues, 41(5), 359-383.

Walsh, R. (2011). Lifestyle and mental health. American Psychologist, 66(7), 579-592.

Weber, R. (2019). Somatic movement dance education: A feminist, cognitive, phenomenological perspective on creativity in dance. In K. Bond, (Ed.), Dance and the quality of life, (pp. 307-324). Cham, Switzerland: Springer. 